\title{
How do Pilon Fractures Heal? An Analysis of Dual Plating, Biomechanically Matched Fixation, and Callus Formation
}

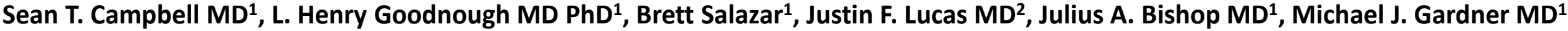

1. Department of Orthopaedic Surgery, Stanford University Medical Center, Stanford, CA, USA 2. Department of Orthopaedic Surgery, Santa Clara Valley Medical Center, San Jose, CA, USA

\section{Background}

- Pilon fixation strategies suggested by recent literature ${ }^{1-3}$ :

1. Dual column/supplemental plate fixation

- Additional support for comminuted metaphysis

- Capture all articular fragments

2. Biomechanically matched fixation

- e.g. medial implant for varus pattern

- Unknown whether supplemental plating augments or impairs healing

- Unclear if biomechanically matched fixation clinically important

- PURPOSE: Determine effect of single vs dual plate and biomechanically matched fixation on metaphyseal callus formation and reoperation rates

\section{Methods}

- Retrospective comparative study

- Single institution, three surgeons

- 50 patients with AO/OTA C2 or C3 (metaphyseal comminution) pilon fractures

- Treated with open reduction and internal fixation, construct at surgeon's discretion

- Modified RUST (mRUST) scores calculated at three and six months post-op

\section{- Compared}

- Single vs dual plate fixation constructs

- Biomechanically matched vs non-matched constructs

- Regression analyses with mRUST >11 and reoperation as dependent variables performed

\section{Figures}

Mean RUST scores at 6 months vs presence of supplemental fixation

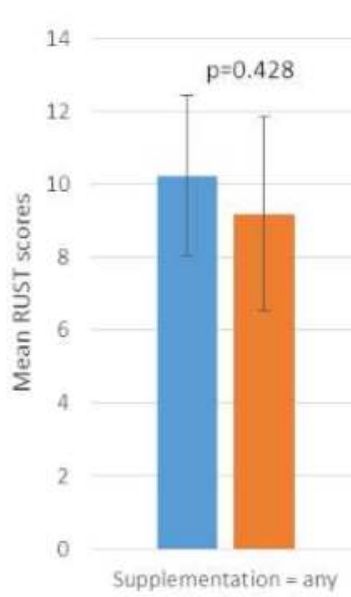

Supplementation $=$ any opposite side fixatio opposite side plate

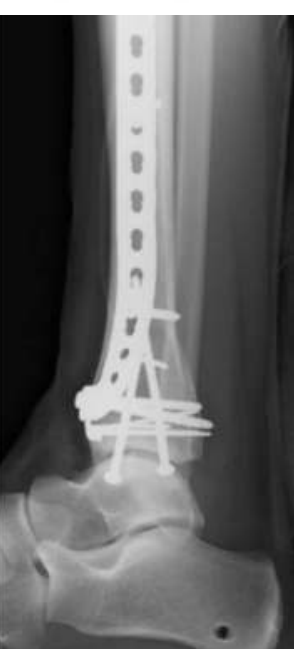

AP and lateral radiographs

demonstrating alternatives to dual metaphyseal pates, including bicortical medial malleolar screws for medial column support and an anterior rim plate to capture articular fragments. White dashed line indicates working length of the construct.

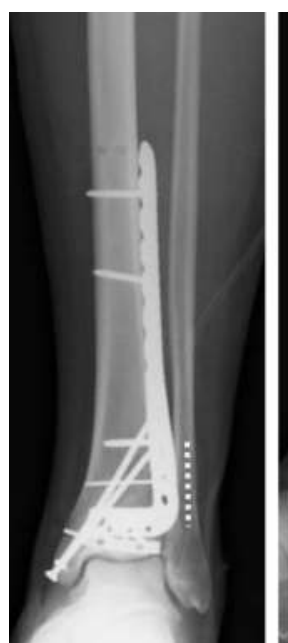

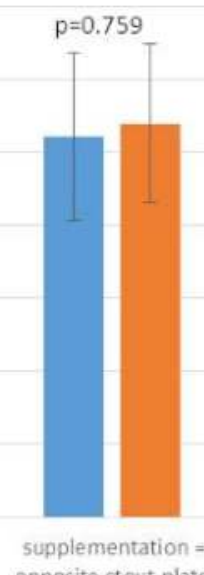

- No supplementation - Supplementatio

\section{Results}

- Six month mRUST scores significantly lower in patients treated with dual plates ( 8.7 vs $10.4, p=0.046$ )

- No difference reoperation rate

- Biomechanically matched fixation did not significantly affect mRUST scores or reoperation rates

- Open fracture risk factor for reoperation (OR 51.1, p=0.008)

- Screw density $0.4-0.5$ protective against reoperation (OR $0.03,0=0.026$ )

\section{Discussion}

- Single plate fixation of pilon fractures

- More metaphyseal callus formation, no difference in reoperation rates

- Future work: Is metaphyseal callus desirable? Are fractures that heal with callus more likely to experience subtle change in alignment or late failure?

\section{References}

Penny P, Swords M, Heisler J, et al. Ability of modern distal tibia plates to stabilize comminuted pilon fracture fragments: Is dual plate fixation necessary? Injury. 2016 Aug;:47(8):1761-1769.

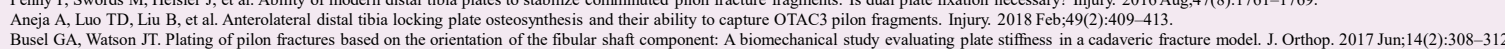

